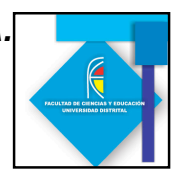

\title{
A PROPÓSITO DE LOS EXPERIMENTOS MENTALES: UNA TENTATIVA PARA LA CONSTRUCCIÓN DE EXPLICACIONES EN CIENCIAS
}

\section{A PURPOSE OF THE THOUGHT EXPERIMENTS: A TENTATIVE EXPLANATION FOR BUILDING SCIENCE}

Yirsen Aguilar $\mathrm{M}^{1}$

Ángel E. Romero ${ }^{2}$

\section{Resumen}

En el análisis realizado en algunas investigaciones se logra evidenciar como el experimento mental se ha constituido, desde la edad media, en una potente estrategia para la construcción de explicaciones científicas. En el siglo XVII se destacan experimentadores mentales, como los de Galileo, Descartes, Newton y Leibniz (Brown, 1986; Koyré, 1968; Kuhn, 1964, 246-252). En los tiempos actuales, la creación de la mecánica cuántica (Kühne, 2005, pp 280-317; Popper, 1959) y la relatividad (Brown, 1987; Norton, 1991 y 1993) son casi impensables sin la función fundamental de los experimentos mentales.

En el año de 1811, en un ensayo Hans Christian Ørsted, es el primero en utilizar el término Gedankenexperiment (experimentos conducidos en los pensamientos) para referirse a una fuente especial de conocimiento ( $c f$. Ørsted, 1811), y fue también el primero en emplear el equivalente alemán Gedankenversuch en 1820. Años más tarde Ernst Mach empleó el término Gedankenexperiment de forma diferente para denotar exclusivamente la conducción imaginaria de un experimento físico real y de allí el contraste entre la experimentación física y el experimento mental. Al respecto se puede decir que fue Ernst Mach quien acuñó este término en la discusión filosófica (Mach, 1897). Este término es utilizado por Mach (1948) en un sentido amplio. Según él, fuera de la experimentación física (empírica), el hombre que llega a un desarrollo intelectual avanzado, recurre a menudo a la experimentación mental. Sin embargo, este tipo de experimento no es usual en la enseñanza de la física.

Con intenciones didácticas se realiza un análisis histórico y epistemológico de la perspectiva Mach (1948) por considerar que su particular manera de asumir estos experimentos, permite recontextualizar estos en la enseñanza de la física. En esta

\footnotetext{
${ }^{1}$ Estudios Culturales sobre las Ciencias y su Enseñanza - ECCE-Facultad de Educación, Universidad de Antioquia. Medellín, Colombia. yirsena@yahoo.es;

${ }^{2}$ Estudios Culturales sobre las Ciencias y su Enseñanza - ECCE-Facultad de Educación, Universidad de Antioquia. Medellín, Colombia.aeromero@ayura.edu.co
} 


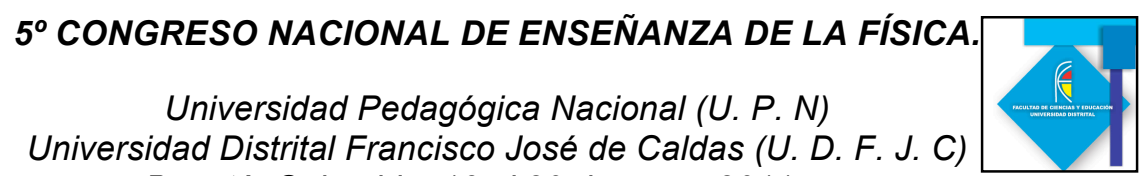

Bogotá, Colombia. 16 al 20 de mayo 2011

perspectiva se asume el experimento mental como la construcción de un escenario hipotético donde se representan circunstancias o eventos que emergen de consecuencias igualmente creadas por el sujeto a partir de conjeturas que responden a una intencionalidad. En términos, el experimento mental puede ser asumido como una acción de pensamiento donde se valoriza la experiencia conservada por el recuerdo y el lenguaje, es decir, el experimento mental se constituye en un recurso de la imaginación que permite crear o visualizar mundos posibles.

A partir del análisis de situaciones clásicas como la caída de los cuerpos en Galileo, El balde con agua en Newton para ilustrar el espacio absoluto, se establecen algunas características, funciones y legitimidad de los experimentos mentales. Finalmente se establecen implicaciones didácticas que permiten ejemplificar experimentos mentales y mostrar que el experimento mental puede ser una tentativa adecuada para la enseñanza de la física.

Palabras clave: Experimento, mental, Galileo, Newton, enseñanza, física y Mach.

\section{Abstract}

In the analysis in some research is done to show how the experiment has been established since the Middle Ages, a powerful strategy for the construction of scientific explanations. In the seventeenth century thought experiments are highlighted, such as Galileo, Descartes, Newton and Leibniz (Brown, 1986; Koyré 1968, Kuhn 1964, 246252). In modern times, the creation of quantum mechanics (Kühne, 2005, pp 280-317, Popper, 1959) and relativity (Brown, 1987; Norton, 1991 and 1993) are almost unthinkable without the crucial role of thought experiments .

In 1811, in an essay Hans Christian Ørsted is the first to use the term Gedankenexperiment (experiments conducted in thought) to refer to a special source of knowledge (cf. Ørsted, 1811), and was also the first Gedankenversuch use the German equivalent in 1820. Years later, Ernst Mach used the term differently Gedankenexperiment to denote only imagined driving a real physical experiment and hence the contrast between physical experiments and the experiment. In this respect we can say that was Ernst Mach who coined this term in the philosophical discussion (Mach, 1897). This term is used by Mach (1948) in a broad sense. According to him, outside the physical testing (empirical), the man who reaches an advanced intellectual development, often uses mental experimentation. However, this type of experiment is unusual in the teaching of physics.

With didactic intentions, an analysis of the historical and epistemological perspective Mach (1948) on the grounds that their particular way of taking these experiments, allows reframing these in the teaching of physics. In this perspective, the experiment is assumed as the construction of a hypothetical scenario where they represent circumstances or events that arise from consequences created by the subject also from conjecture to respond to an intentionality. In terms, the experiment can be assumed as an act of 


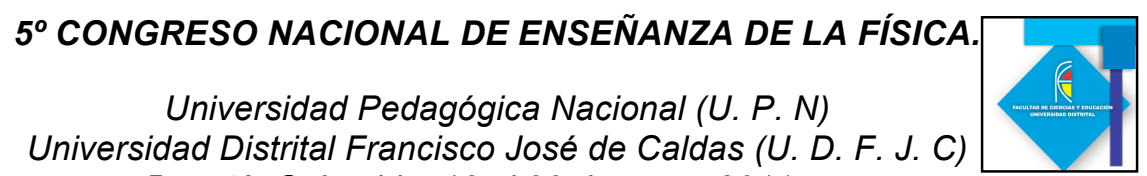

Bogotá, Colombia. 16 al 20 de mayo 2011

thought which values the experience preserved for the memory and language, ie, the thought experiment becomes a resource of imagination for creating and viewing possible worlds.

From the analysis of classic situations like the fall of bodies in Galileo, the bucket of water to illustrate Newton's absolute space, sets some features, functions and legitimacy of the experiments. Educational implications are finally established that thought experiments can illustrate and experiment show that an attempt may be suitable for physics education.

Keywords: Experiment, mental, Galileo, Newton, education, physical and Mach.

\section{Conceptualización sobre el experimento mental}

En el año de 1811, en un ensayo Hans Christian Ørsted, es el primero en utilizar el término Gedankenexperiment (experimentos conducidos en los pensamientos) para referirse a una fuente especial de conocimiento (cf. Ørsted, 1811), y fue también el primero en emplear el equivalente alemán Gedankenversuch en 1820. Años más tarde Ernst Mach empleó el término Gedankenexperiment de forma diferente para denotar exclusivamente la conducción imaginaria de un experimento físico real y de allí el contraste entre la experimentación física y el experimento mental. Al respecto se puede decir que fue Ernst Mach quien acuñó este término en la discusión filosófica (Mach, 1897). Este término es utilizado por Mach (1948) en un sentido amplio. Según él, fuera de la experimentación física (empírica), el hombre que llega a un desarrollo intelectual avanzado, recurre a menudo a la experimentación mental.

Aquellos que hacen proyectos, aquellos que construyen castillos, romanceros $y$ poetas que se dejan llevar por utopías sociales o técnicas, hacen experimentación mental; la hacen también el comerciante serio, el inventor reflexivo y el sabio.

Todos se representan circunstancias diversas y relacionan a estas representaciones ciertas conjeturas. Pero los primeros cambian en su imaginación circunstancias que no se encuentran en la realidad, o bien se representan estas circunstancias seguidas de consecuencias que no tienen vínculos con ellas, mientras que el comerciante, el inventor y el sabio tienen como representaciones buenas imágenes de los hechos y permanecen en sus pensamientos muy próximos a la realidad (Mach, 1948, p. 158)

Bajo estas circunstancias, ¿Qué se puede entender como experimento metal? Los experimentos mentales, al igual que los experimentos físicos reales, se constituyen en un método para construir, validar, profundizar y contrastar teorías o explicaciones. Pero los experimentos mentales tienen, a demás la importante función de ser una precondición necesaria para planear y ejecutar experimentos reales. Sin embargo, es oportuno precisar que aunque se trata de una investigación imaginaria que no necesariamente debe ser ejecutada en el mundo físico real, el experimento mental está sujeto a ciertas exigencias teóricas que comparte con los experimentos reales. En este sentido, se puede decir que en un experimento mental, al igual que en el experimento físico real, se estudia la dependencia funcional de las variables planeando y controlando cambios de datos. 


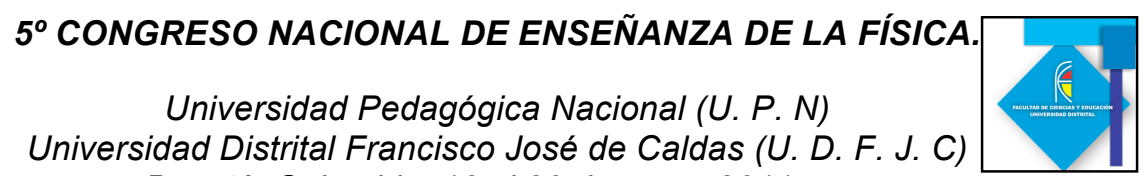

Bogotá, Colombia. 16 al 20 de mayo 2011

Además, todos los experimentos mentales, tal como ocurre en los experimentos físicos reales, dependen de ciertas suposiciones y teorías de trasfondo.

En este orden de ideas, la experimentación mental puede ser considerada como la construcción de un escenario hipotético donde se representan circunstancias o eventos que emergen de consecuencias igualmente creadas por el sujeto a partir de conjeturas que responden a intencionalidades. En estos términos, el experimento mental puede ser asumido como una acción de pensamiento donde se valoriza la experiencia conservada por el recuerdo y el lenguaje, es decir, el experimento mental se constituye en un recurso de la imaginación que permite crear o visualizar mundos posibles.

A partir de estas consideraciones, una descripción aproximada a lo que caracteriza la ejecución de un experimento mental es la siguiente: una vez identificado el sistema y se hagan emerger las variables (circunstancias), se hacen variar todas estas circunstancias que influyen en el resultado, es decir, se imagina una variación continua que pase revista a todos los casos posibles. De esta manera, si se pudiera precisar las características de la ejecución de un experimento mental, se diría que es aplicar un método variacional teniendo en cuenta la continuidad de las circunstancias y la adaptación de pensamientos.

\section{Funciones y características del experimento mental}

Algunos experimentos mentales permiten la profundización de teorías, es el caso del experimento mental que realiza Galileo sobre la inercia. Otros se realizan porque es imposible ejecutar el escenario de la experimentación en el mundo real, por ejemplo el observador de Einstein que viaja en un rayo de luz. Igualmente, los experimentos mentales ayudan a explicar y aclarar los estados abstractos de las cosas, facilitando así el proceso de comprensión, un ejemplo es el experimento que realiza Newton del balde con agua para ilustrar el espacio absoluto. Otros sirven como ejemplo en el análisis conceptual. Una característica es la flexibilidad de los experimentos mentales, ya que estos son repensados de modo que se puede realizar una versión diferente del mismo escenario para contrastar los postulados situados. Estos experimentos pueden evolucionar y ser objetos de modificaciones con el tiempo. Que los experimentos mentales puedan ser repensados cohesiona con otra característica relacionada con la "importancia histórica y probatoria a nivel local, es decir, cuando y donde los locales que atribuyen importancia probatoria a él [...] los apruebe." (McAllister, 1996, p. 248).

En términos generales puede decirse que:

i) Los experimentos mentales se caracterizan porque alcanzan sus propósitos sin necesidad de ser ejecutados en un experimento físico real.

ii) Los experimentos mentales son experimentos en tanto que comparten ciertos requisitos teóricos mínimos con los experimentos reales, tales como: a) el cambio planeado y controlado de datos; b) mostrar la manera en que en una situación artificial las variables dependen funcionalmente unas de otras; c) la dependencia de ciertas hipótesis y teorías de trasfondo con miras al análisis y la evaluación del argumento. 


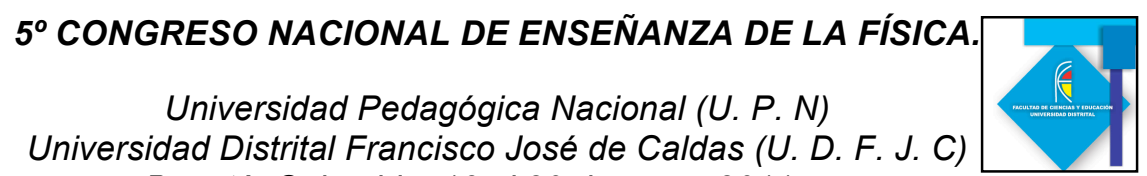

Universidad Distrital Francisco José de Caldas (U.
Bogotá, Colombia. 16 al 20 de mayo 2011

iii) Los experimentos mentales dependen de ciertas suposiciones y teorías que sustentan las conjeturas y asociaciones que hace el sujeto, es decir, la facultad intuitiva como vehículo en un experimento mental.

iv) Entre las funciones y propósitos de los experimentos mentales se destaca: a) probar que ciertas teorías o conceptos envuelven contradicciones; b) proporcionar evidencia de respaldo a una teoría o un concepto; c) ilustrar una posición abstracta o compleja; d) detectar la vaguedad de un concepto y sus casos dudosos de aplicación (Brendel, 2003). Pero también se puede decir que a partir de los experimentos mentales se realizan construcciones que se constituyen en condiciones iníciales necesarias para la construcción de un marco teórico, tal como sucede con el espacio absoluto y el tiempo absoluto en Newton.

\section{Implicaciones didácticas}

En la experimentación mental puede ocurrir que no se llegue a lo que está más conforme con lo que se conoce, por ejemplo, algunos estudiantes consideran que duplicando el lado de un cuadrado también se duplica la superficie, consideran también que al duplicar la longitud de un péndulo también se duplica el período. El método que consiste en adivinar una serie de experiencias tiene un alto valor didáctico. Si bien en estas aseveraciones se identifican dificultades de orden conceptual permiten captar bien la diferencia entre lo que es determinado lógicamente o físicamente, y lo que resulta de una pura analogía; enseñan también a distinguir lo que puede ser adivinado de aquello que absolutamente no puede serlo (Mach, 1948. p 167).

Se puede decir entonces que, si bien la experimentación mental es suprema mente importante para el hombre de ciencia profesional, es también importante y necesario para el desarrollo psíquico del individuo y para aquel estudiante que aprende Ciencias.

Tan importantes han sido los experimentos prácticos como los mentales en el desarrollo de la Física, que bien vale la pena ejercitar a los estudiantes en los dos tipos de prácticas. De cualquier manera el experimento práctico requiere de un cierto ejercicio mental y a su vez un experimento mental requiere de ciertas referencias desde la práctica, formando una llave que no se puede disolver, el experimento mental resulta particularmente útil en el desarrollo de la clase teórica, puesto que obliga a viajar por el pensamiento con imaginación y creatividad. La importancia de esta forma de proceder como un elemento apropiado para la producción científica fue reconocida por personajes como Einstein (Einstein, 1986).

El principio de inercia marca, en realidad, el verdadero comienzo de la Física. Fue adquirido, como sabemos, imaginando el experimento ideal de un cuerpo en movimiento perenne, sin rozamiento ni bajo la acción de ninguna otra fuerza exterior. Con este ejemplo y después con otros más, hemos podido aquilatar la importancia de la introducción del experimento ideal... Aunque estos puedan parecer demasiado fantásticos, nos ayudarán sin embargo, a comprender todo lo que se pueda de la 


\section{$5^{\circ}$ CONGRESO NACIONAL DE ENSEÑANZA DE LA FÍSICA}

Universidad Pedagógica Nacional (U. P. N)

Universidad Distrital Francisco José de Caldas (U. D. F. J. C)

Bogotá, Colombia. 16 al 20 de mayo 2011

teoría de la relatividad, con las limitaciones inherentes a los métodos simples que estamos utilizando.

\section{Referencias Bibliográficas:}

Brendel, E. "Pompas de intuición y el uso adecuado de los experimentos mentales", en: Ideas y Valores, CXXIII (2003), pp. 3-23.

Brown, J. R. (1991a). The Laboratory of the Mind. London/New York: Routledge.(1991b). "Thought Experiments: A Platonic Account". En: Horowitz \&Massey (eds.), Thought Experiments in Science and Philosoph.y. Savage: Rowman \& Littlefield.

Brown, James R., 1986, "experimentos mentales desde la Revolución Científica", Estudios Internacionales en la Filosofía de la Ciencia, 1: 1-15.

Einstein, A. (1986). La evolución de la física, Editorial Salvat. Barcelona.

Koyré, A (1968). Metaphysics and Measurement, London: Chapman and Hall.

Kuhn, T. (1964). "Una función de los experimentos mentales", reimpreso en T.

Kühne, Ulrich, 2005, Die-des-Gedankenexperiments Methode, Frankfurt: Suhrkamp.

McAllister, James, 1996, "The Evidential Significance of Thought Experiments in Science", Studies in History and Philosophy of Science, 27: 233-250.

Mach, E. (1948). Conocimiento y Error. Espasa. Argentina.

Mach, E. (1897). "Über Gedankenexperimente". En: Zeitschrift für physikalischen. und chemischen Unterricht vol. 10, 1-5. (1905). Erkenntnis und Irrtum. Leipzig: editorial 18097.

Norton, J. D. (1991). "experimentos mentales en el trabajo de Einstein", en T. Horowitz y G. Massey (eds.), los experimentos mentales de la Ciencia y Filosofía,Lanham: Rowman \& Littlefield, 129-148.

Ørsted, H. C. (1811). Förste Indledning til den Almindelige Naturlae- re, et indbydelsesskrivt til forelaesninger over denne vindenskab, Copenha-gen. [Traducción alemana (1822). „Über Geist und Studium der allge-meinen Naturlehre”. En: Gehlens Journal für Chemie und Physik vol. 36:458-88]

Popper, K. (1959). "Sobre el uso y mal uso de los experimentos imaginarios, especialmente en la Teoría Cuántica", en La lógica del descubrimiento científico, Londres: Hutchinson, 442-456. 